\title{
Weed Control in White Bean with Various Halosulfuron Tankmixes
}

\author{
Nader Soltani, ${ }^{1}$ Robert E. Nurse, ${ }^{2}$ Christy Shropshire, ${ }^{1}$ and Peter H. Sikkema ${ }^{1}$ \\ ${ }^{1}$ University of Guelph Ridgetown Campus, 120 Main Street East, Ridgetown, ON, Canada NOP 2C0 \\ ${ }^{2}$ Agriculture and Agri-Food Canada, Harrow, ON, Canada NOR $1 G 0$ \\ Correspondence should be addressed to Nader Soltani; soltanin@uoguelph.ca
}

Received 16 July 2014; Accepted 6 October 2014; Published 27 October 2014

Academic Editor: Albino Maggio

Copyright (C) 2014 Nader Soltani et al. This is an open access article distributed under the Creative Commons Attribution License, which permits unrestricted use, distribution, and reproduction in any medium, provided the original work is properly cited.

Four field trials were conducted over a three-year period (2011-2013) in southwestern Ontario to evaluate the level of weed control provided by various halosulfuron tankmixes applied preplant incorporated (PPI) in white bean. Trifluralin, s-metolachlor, halosulfuron, and imazethapyr applied alone or in combination caused $4 \%$ or less visible injury 1 and 4 weeks after emergence (WAE) in white bean. Trifluralin, s-metolachlor, halosulfuron, and imazethapyr applied PPI provided 80-96\%, 84-95\%, 83-100\%, and $75-92 \%$ control of redroot pigweed; $19-28 \%, 30-40 \%, 97-99 \%$, and $73-84 \%$ control of common ragweed; 94-96\%, 63-82\%, 96-100\%, and $96-100 \%$ control of common lambsquarters; $14-15 \%, 12-35 \%, 100 \%$, and $96-97 \%$ control of wild mustard; and $96-$ $97 \%, 95-97 \%, 53-56 \%$, and $80-82 \%$ control of green foxtail, respectively. The two- and three-way tankmixes of halosulfuron with trifluralin, s-metolachlor, or imazethapyr provided $85-100 \%$ control of redroot pigweed, $90-98 \%$ control of common ragweed, $97-$ $100 \%$ control of common lambsquarters, $100 \%$ control of wild mustard, and 93-98\% control of green foxtail. Weed density, weed biomass and white bean seed yields reflected the level of visible weed control.

\section{Introduction}

Ontario is one of the main regions for the production of white (navy) bean (Phaseolus vulgaris L.) in North America. In 2012, white bean growers in Ontario planted 28,000 hectares and produced 68,000 MT of white bean with a farm-gate value of approximately $\$ 38$ million [1]. White bean has short physical stature and therefore is more vulnerable to weed interference and subsequent yield losses if weeds are not adequately controlled [2-6]. Yield losses of 59\% have been attributed to weed interference in dry beans in Ontario which is substantially greater than other field crops such as winter wheat (3\%), spring cereals (12\%), soybean (38\%), and corn (52\%) [7]. There is only one registered soil applied broadleaf herbicide, imazethapyr, for weed control in white bean in Ontario. Imazethapyr provides only marginal control of common lambsquarters (Chenopodium album L.) and common ragweed (Ambrosia artemesiifolia L.) and has a narrow margin of crop safety in dry bean $[2,6]$. More research is needed to identify new herbicide options for annual broadleaf weed management in white bean.
Halosulfuron is a newly registered sulfonylurea herbicide that controls redroot pigweed (Amaranthus retroflexus L.), common lambsquarters, common ragweed, wild mustard (Sinapis arvensis L.), nutsedge species (Cyperus spp.), ladysthumb (Polygonum persicaria L.), velvetleaf (Abutilon theophrasti Medic.), and cocklebur (Xanthium strumarium L.), including glyphosate and triazine resistant biotypes [8]. However, halosulfuron is weak on controlling troublesome annual grasses such as Setaria, Digitaria, Echinochloa, and Panicum species.

Trifluralin is a dinitroaniline herbicide that controls several annual grasses including Setaria, Digitaria, Echinochloa, and Panicum spp. and some broadleaved weeds such as common lambsquarters and redroot pigweed, including acetolactate synthase and triazine resistant biotypes [7].

S-metolachlor is a chloroacetanilide herbicide that controls annual grasses such as green foxtail (Setaria viridis (L.) Beauv.), yellow foxtail (Setaria glauca (L.) Beauv.), giant foxtail (Setaria faberii Herrm.), smooth crabgrass (Digitaria ischaemum (Schreb) Muhl.), large crabgrass (Digitaria sanguinalis (L.) Scop.), barnyardgrass (Echinochloa crusgalli (L.) 
TABLE 1: Percent visible injury 1 and 4 WAE, crop moisture at harvest, and yield of white bean treated with halosulfuron tankmixes applied PPI at Exeter (2011-2013) and Harrow, ON (2012). Means followed by the same letter within a column are not significantly different according to Fisher's Protected LSD at $P<0.05^{\mathrm{a}}$.

\begin{tabular}{|c|c|c|c|c|c|}
\hline \multirow{3}{*}{ Herbicide treatment } & \multirow{3}{*}{ Rate (g ai ha ${ }^{-1}$ ) } & \multicolumn{2}{|c|}{ Injury } & \multirow{3}{*}{ Moisture } & \multirow{3}{*}{$\begin{array}{l}\text { Yield } \\
\text { Tha }^{-1}\end{array}$} \\
\hline & & $1 \mathrm{WAE}$ & 4 WAE & & \\
\hline & & & $\%$ & & \\
\hline Weedy check & & 0 & $0 \mathrm{a}$ & 20.8 & $0.6 \mathrm{e}$ \\
\hline Weed-free check & & 0 & $0 \mathrm{a}$ & 19.9 & $2.2 \mathrm{ab}$ \\
\hline trifluralin & 600 & 0 & $0 \mathrm{a}$ & 20.3 & $1.2 \mathrm{~d}$ \\
\hline s-Metolachlor & 1050 & 0 & $0 \mathrm{a}$ & 20.4 & $1.4 \mathrm{~cd}$ \\
\hline Halosulfuron & 35 & 0 & $0 \mathrm{a}$ & 19.6 & $1.7 \mathrm{bc}$ \\
\hline Imazethapyr & 45 & 0 & $2 \mathrm{bc}$ & 19.9 & $1.8 \mathrm{bc}$ \\
\hline Trifluralin + halosulfuron & $600+35$ & 0 & $0 \mathrm{a}$ & 19.4 & $2.2 \mathrm{ab}$ \\
\hline Trifluralin + imazethapyr & $600+45$ & 0 & $2 \mathrm{bc}$ & 19.5 & $2.0 \mathrm{ab}$ \\
\hline Trifluralin + halosulfuron + imazethapyr & $600+35+45$ & 0 & $4 \mathrm{c}$ & 19.5 & $2.1 \mathrm{ab}$ \\
\hline s-Metolachlor + halosulfuron & $1050+35$ & 0 & $0 \mathrm{a}$ & 19.6 & $2.2 \mathrm{ab}$ \\
\hline s-Metolachlor + imazethapyr & $1050+45$ & 0 & $1 \mathrm{~b}$ & 19.8 & $2.0 \mathrm{ab}$ \\
\hline s-Metolachlor + halosulfuron + imazethapyr & $1050+35+45$ & 0 & $3 \mathrm{bc}$ & 19.9 & $2.0 \mathrm{ab}$ \\
\hline
\end{tabular}

a Abbreviations: WAE, weeks after crop emergence; PPI, preplant incorporated.

Beauv.), fall panicum (Panicum dichotomiflorum Michx.), and witchgrass (Panicum capillare L.) $[7,8]$ and some annual broadleaf weeds such as redroot pigweed and Eastern black nightshade (Solanum ptycanthum Dunal).

Tankmixing halosulfuron with trifluralin, S-metolachlor, and/or imazethapyr can provide Ontario white bean growers with a herbicide option that provides full-season control of annual grass and broadleaf weeds. Utilization of herbicide tankmixes will also reduce the selection pressure for herbicide resistant weeds.

There is little information on the crop safety and efficacy of halosulfuron tankmixed with trifluralin, s-metolachlor, and/or imazethapyr applied preplant incorporated (PPI) for the control of annual grass and broadleaf weeds under Ontario environmental conditions. The objective of this research was to evaluate the level of crop injury and weed control provided by the two- and three-way tankmixes of halosulfuron with trifluralin, s-metolachlor, and/or imazethapyr applied PPI in white bean.

\section{Materials and Methods}

Field studies were conducted in 2011, 2012, and 2013 at the Huron Research Station, University of Guelph, Exeter, Ontario, and in 2012 at the Agriculture and Agri-Food Canada Research Centre, Harrow, Ontario. The soil at Exeter was a Brookston clay loam (Orthic Humic Gleysol, mixed, mesic, and poorly drained) with $32 \%$ sand, $42 \%$ silt, $26 \%$ clay, $3.7 \%$ organic matter, and $\mathrm{pH} 7.8$ in $2011 ; 41 \%$ sand, $35 \%$ silt, $24 \%$ clay, $3.2 \%$ organic matter, and pH 7.9 in 2012; and $29 \%$ sand, $44 \%$ silt, $27 \%$ clay, $3.6 \%$ organic matter, and $\mathrm{pH} 7.7$ in 2013. The soil at Harrow was a Fox sandy loam (Brunisolic Gray Brown Luvisol) with $82.5 \%$ sand, 5\% silt, $12.5 \%$ clay, $2.6 \%$ organic matter, and $\mathrm{pH}$ of 6.0 . Seedbed preparation at all sites consisted of fall moldboard plowing followed by two passes with a field cultivator with rolling basket harrows in the spring.
The experiments were arranged in a randomized complete block design (RCBD) with four replications. There were twelve treatments as listed in Table 1. Plots consisted of four rows of white bean "T9905" planted at a rate of 250,000 seeds $\mathrm{ha}^{-1}$ to a depth of $5 \mathrm{~cm}$ in rows that were spaced $0.75 \mathrm{~m}$ apart and were 8 or $10 \mathrm{~m}$ long in late May to early June of each year.

Herbicide treatments were made with a $\mathrm{CO}_{2}$-pressurized backpack sprayer calibrated to deliver $200 \mathrm{~L} \mathrm{ha}^{-1}$ of spray solution at a pressure of $200 / 240 \mathrm{kPa}$ using low drift nozzles (ULD120-02, Spraying Systems Co., P.O. Box 7900. Wheaton, IL). The boom was $1.5 \mathrm{~m}$ wide with four nozzles spaced $0.5 \mathrm{~m}$ apart. PPI herbicides were applied 1-2 days before planting and were immediately incorporated. Weed-free check plots were maintained with interrow cultivation and hand hoeing during the growing season.

White bean injury and weed control were visually estimated on a scale of 0 (no injury/control) to $100 \%$ (complete plant death). White bean injury was assessed 1 and 4 weeks after emergence (WAE). Weed control was assessed 4 and 8 WAE, respectively. Weed density and dry weight were evaluated 8 WAE by counting and cutting plants at the soil surface from two $0.5 \mathrm{~m}^{2}$ quadrats per plot and separating by species. Plants were dried at $60 \mathrm{C}$ to a constant moisture (at least for 48 hours) and then weighed. White bean was considered mature when $90 \%$ of the pods in the weed-free check had turned from green to a golden colour. Beans were harvested from the two center rows of each plot with a small plot combine, weight and seed moisture content were recorded, and seed yields were adjusted to $18 \%$ seed moisture content.

Data were analyzed as an RCBD using PROC MIXED in SAS 9.2. Herbicide treatment was considered a fixed effect, while environment (year-location combinations), the interaction between environment and herbicide treatment, and replicate nested within environment were considered random effects. Significance of the fixed effect was tested 
TABLE 2: Percent control, density, and dry weight of redroot pigweed in white bean treated with halosulfuron tankmixes applied PPI at Exeter (2011-2012) and Harrow, ON (2012). Means followed by the same letter within a column are not significantly different according to Fisher's Protected LSD at $P<0.05^{\mathrm{a}}$.

\begin{tabular}{|c|c|c|c|c|c|}
\hline \multirow{3}{*}{ Herbicide treatment } & \multirow{3}{*}{ Rate (g ai ha ${ }^{-1}$ ) } & \multicolumn{2}{|c|}{ Control } & \multirow{3}{*}{$\begin{array}{l}\text { Density } \\
\# \mathrm{~m}^{-2}\end{array}$} & \multirow{3}{*}{$\begin{array}{c}\text { Dry weight } \\
\mathrm{g} \mathrm{m}^{-2}\end{array}$} \\
\hline & & 4 WAE & 8 WAE & & \\
\hline & & \multicolumn{2}{|c|}{$\%$} & & \\
\hline Weedy check & & 0 & 0 & $6.0 \mathrm{c}$ & $29.7 \mathrm{c}$ \\
\hline Weed-free check & & 100 & 100 & $0.0 \mathrm{a}$ & $0.0 \mathrm{a}$ \\
\hline Trifluralin & 600 & 80 & 96 & $0.7 \mathrm{ab}$ & $4.9 \mathrm{~b}$ \\
\hline s-Metolachlor & 1050 & 84 & 95 & $0.6 \mathrm{a}$ & $4.3 \mathrm{ab}$ \\
\hline Halosulfuron & 35 & 83 & 100 & $0.8 \mathrm{ab}$ & $2.5 \mathrm{ab}$ \\
\hline Imazethapyr & 45 & 75 & 92 & $2.0 \mathrm{~b}$ & $3.9 \mathrm{ab}$ \\
\hline Trifluralin + halosulfuron & $600+35$ & 85 & 100 & $0.6 \mathrm{a}$ & $3.3 \mathrm{ab}$ \\
\hline Trifluralin + imazethapyr & $600+45$ & 78 & 100 & $0.8 \mathrm{ab}$ & $5.8 \mathrm{~b}$ \\
\hline Trifluralin + halosulfuron + imazethapyr & $600+35+45$ & 91 & 100 & $0.6 \mathrm{a}$ & $3.5 \mathrm{ab}$ \\
\hline s-Metolachlor + halosulfuron & $1050+35$ & 87 & 100 & $0.4 \mathrm{a}$ & $2.0 \mathrm{ab}$ \\
\hline s-Metolachlor + imazethapyr & $1050+45$ & 87 & 100 & $0.3 \mathrm{a}$ & $1.6 \mathrm{ab}$ \\
\hline s-Metolachlor + halosulfuron + imazethapyr & $1050+35+45$ & 91 & 100 & $0.1 \mathrm{a}$ & $0.3 \mathrm{a}$ \\
\hline
\end{tabular}

abbreviations: WAE, weeks after crop emergence; PPI, preplant incorporated.

using $F$-test and random effects were tested using a $Z$ test of the variance estimate. The UNIVARIATE procedure was used to test data for normality and homogeneity of variance. For all ratings, any treatments assigned a value of zero (weedy and weed-free checks for crop injury; weedy check for weed control; weed-free check for weed density and dry weight) were excluded from the analysis. However, all values were compared independently to zero to evaluate treatment differences with the weedy and/or weed-free check. To satisfy the assumptions of the variance analyses, injury 1 and 4 WAE and weed control were arcsine square root transformed and weed density, dry weight, and crop moisture were $\log$ transformed. Treatment comparisons were made using Fisher's Protected LSD at a level of $P<0.05$. Data compared on the transformed scale were converted back to the original scale for presentation of results.

\section{Results and Discussion}

3.1. Crop Injury. The analysis of variance (ANOVA) indicated no effect of years or treatment by year interaction for crop injury; therefore, data were pooled and averaged for environments.

Trifluralin, s-metolachlor, halosulfuron, and imazethapyr applied alone or in combination applied PPI caused $4 \%$ or less visible injury 1 and 4 weeks after emergence (WAE) in white bean (Table 1). This is similar to other studies that have shown up to $6 \%$ injury in white bean with halosulfuron applied alone or in tankmixes with other herbicides [9-11]. Silvey et al. [12] also found minimal and transient injury (5\%) with halosulfuron in snap bean. In other studies, halosulfuron reduced yield of white bean as much as $9 \%$ and adzuki bean as much as $68 \%$ but had no effect on the seed yield of black, cranberry, kidney, otebo, pinto, and small red Mexican beans $[9,10]$. In another study halosulfuron applied postemergence reduced seed yield of white bean as much as $21 \%$ [11].
However, other studies have shown significant injury with halosulfuron alone or in tankmixes with other herbicides in adzuki bean [10]. There was no difference in seed moisture content between treatments evaluated (Table 1).

All treatments increased white bean seed yield compared to the weedy check (Table 1). Weed interference with trifluralin, s-metolachlor, halosulfuron, and imazethapyr applied alone resulted in a decrease in white bean seed yield of 45,36 , 23 , and $18 \%$ compared to the weed-free check, respectively (Table 1). In contrast, the level of weed control with trifluralin + halosulfuron, trifluralin + imazethapyr, trifluralin + halosulfuron + imazethapyr, s-metolachlor + halosulfuron, s-metolachlor + imazethapyr, and s-metolachlor + halosulfuron + imazethapyr at rates evaluated resulted in white bean seed yield that was equivalent to the weed-free check (Table 1).

3.2. Weed Control. The dominant weed species in this study were redroot pigweed, common ragweed, common lambsquarters, wild mustard, and green foxtail. The ANOVA indicated no effect of environments or treatment by environments for weed control; therefore, data were pooled and averaged for environments (Tables 2-6).

3.2.1. Redroot Pigweed. Trifluralin, s-metolachlor, halosulfuron, imazethapyr, trifluralin + halosulfuron, trifluralin + imazethapyr, trifluralin + halosulfuron + imazethapyr, smetolachlor + halosulfuron, s-metolachlor + imazethapyr, and s-metolachlor + halosulfuron + imazethapyr applied PPI controlled redroot pigweed $80-96 \%, 84-95 \%, 83-100 \%$, 75$92 \%, 85-100 \%, 78-100 \%, 91-100 \%, 87-100 \%, 87-100 \%$, and 91-100\%, (Table 2).

Trifluralin, s-metolachlor, halosulfuron, and imazethapyr applied PPI reduced redroot pigweed density $67-90 \%$ and biomass $84-92 \%$ (Table 2). Trifluralin + halosulfuron, trifluralin + imazethapyr, trifluralin + halosulfuron + imazethapyr, 
TABLE 3: Percent control, density, and dry weight of common ragweed in white bean treated with halosulfuron tankmixes applied PPI at Exeter, ON (2012-2013). Means followed by the same letter within a column are not significantly different according to Fisher's Protected LSD at $P<0.05^{\mathrm{a}}$.

\begin{tabular}{|c|c|c|c|c|c|}
\hline \multirow{3}{*}{ Herbicide treatment } & \multirow{3}{*}{ Rate (g ai ha ${ }^{-1}$ ) } & \multicolumn{2}{|c|}{ Control } & \multirow{3}{*}{$\begin{array}{l}\text { Density } \\
\# \mathrm{~m}^{-2}\end{array}$} & \multirow{3}{*}{$\begin{array}{c}\text { Dry weight } \\
\mathrm{g} \mathrm{m}^{-2}\end{array}$} \\
\hline & & $4 \mathrm{WAE}$ & 8 WAE & & \\
\hline & & \multicolumn{2}{|c|}{$\%$} & & \\
\hline Weedy check & & $0 \mathrm{e}$ & $0 \mathrm{e}$ & $1.8 \mathrm{~b}$ & $5.2 \mathrm{bc}$ \\
\hline Weed-free check & & $100 \mathrm{a}$ & $100 \mathrm{a}$ & $0.0 \mathrm{a}$ & $0.0 \mathrm{a}$ \\
\hline Trifluralin & 600 & $28 \mathrm{~d}$ & $19 \mathrm{e}$ & $2.7 \mathrm{~b}$ & $6.6 \mathrm{c}$ \\
\hline s-Metolachlor & 1050 & $30 \mathrm{~d}$ & $40 \mathrm{~d}$ & $2.5 \mathrm{~b}$ & $18.6 \mathrm{c}$ \\
\hline Halosulfuron & 35 & 99 a & $97 \mathrm{ab}$ & $0.1 \mathrm{a}$ & $0.0 \mathrm{a}$ \\
\hline Imazethapyr & 45 & $84 \mathrm{c}$ & $73 \mathrm{c}$ & $1.2 \mathrm{ab}$ & $1.7 \mathrm{ab}$ \\
\hline Trifluralin + halosulfuron & $600+35$ & $96 \mathrm{ab}$ & $90 \mathrm{abc}$ & $0.3 \mathrm{a}$ & $0.6 \mathrm{ab}$ \\
\hline Trifluralin + imazethapyr & $600+45$ & $88 \mathrm{c}$ & $78 \mathrm{bc}$ & $1.0 \mathrm{ab}$ & $2.3 \mathrm{ab}$ \\
\hline Trifluralin + halosulfuron + imazethapyr & $600+35+45$ & $98 \mathrm{a}$ & $96 \mathrm{ab}$ & $0.0 \mathrm{a}$ & $0.0 \mathrm{a}$ \\
\hline s-Metolachlor + halosulfuron & $1050+35$ & $98 \mathrm{a}$ & $93 \mathrm{abc}$ & $0.2 \mathrm{a}$ & $0.3 \mathrm{ab}$ \\
\hline s-Metolachlor + imazethapyr & $1050+45$ & $90 \mathrm{bc}$ & $78 \mathrm{bc}$ & $0.9 \mathrm{ab}$ & $1.2 \mathrm{ab}$ \\
\hline s-Metolachlor + halosulfuron + imazethapyr & $1050+35+45$ & $98 \mathrm{a}$ & $94 \mathrm{ab}$ & $0.0 \mathrm{a}$ & $0.0 \mathrm{a}$ \\
\hline
\end{tabular}

${ }^{a}$ Abbreviations: WAE, weeks after crop emergence; PPI, preplant incorporated.

TABLE 4: Percent control, density, and dry weight of common lambsquarters in white bean treated with halosulfuron tankmixes applied PPI at Exeter (2011-2013) and Harrow, ON (2012). Means followed by the same letter within a column are not significantly different according to Fisher's Protected LSD at $P<0.05^{\mathrm{a}}$.

\begin{tabular}{|c|c|c|c|c|c|}
\hline \multirow{3}{*}{ Herbicide treatment } & \multirow{3}{*}{ Rate (g ai ha ${ }^{-1}$ ) } & \multicolumn{2}{|c|}{ Control } & \multirow{3}{*}{$\begin{array}{l}\text { Density } \\
\# \mathrm{~m}^{-2}\end{array}$} & \multirow{3}{*}{$\begin{array}{c}\text { Dry weight } \\
\mathrm{g} \mathrm{m}^{-2}\end{array}$} \\
\hline & & $4 \mathrm{WAE}$ & $8 \mathrm{WAE}$ & & \\
\hline & & \multicolumn{2}{|c|}{$\%$} & & \\
\hline Weedy check & & $0 \mathrm{~d}$ & $0 \mathrm{~d}$ & $6.8 c$ & $9.3 \mathrm{~b}$ \\
\hline Weed-free check & & $100 \mathrm{a}$ & $100 \mathrm{a}$ & $0.0 \mathrm{a}$ & $0.0 \mathrm{a}$ \\
\hline Trifluralin & 600 & $94 \mathrm{bc}$ & $96 \mathrm{~b}$ & $0.5 \mathrm{ab}$ & $1.4 \mathrm{a}$ \\
\hline s-Metolachlor & 1050 & $82 \mathrm{c}$ & $63 c$ & $3.9 c$ & $12.7 \mathrm{~b}$ \\
\hline Halosulfuron & 35 & $96 \mathrm{ab}$ & $100 \mathrm{a}$ & $0.5 \mathrm{ab}$ & $1.4 \mathrm{a}$ \\
\hline Imazethapyr & 45 & $96 \mathrm{ab}$ & $100 \mathrm{a}$ & $1.3 \mathrm{~b}$ & $2.2 \mathrm{a}$ \\
\hline Trifluralin + halosulfuron & $600+35$ & $97 \mathrm{ab}$ & $100 \mathrm{a}$ & $0.4 \mathrm{ab}$ & $1.7 \mathrm{a}$ \\
\hline Trifluralin + imazethapyr & $600+45$ & $96 \mathrm{ab}$ & $100 \mathrm{a}$ & $0.4 \mathrm{ab}$ & $1.1 \mathrm{a}$ \\
\hline Trifluralin + halosulfuron + imazethapyr & $600+35+45$ & $99 \mathrm{ab}$ & $100 \mathrm{a}$ & $0.2 \mathrm{a}$ & $0.5 \mathrm{a}$ \\
\hline s-Metolachlor + halosulfuron & $1050+35$ & $99 \mathrm{ab}$ & $100 \mathrm{a}$ & $0.2 \mathrm{a}$ & $1.6 \mathrm{a}$ \\
\hline s-Metolachlor + imazethapyr & $1050+45$ & $98 \mathrm{ab}$ & $100 \mathrm{a}$ & $0.3 \mathrm{ab}$ & $0.8 \mathrm{a}$ \\
\hline s-Metolachlor + halosulfuron + imazethapyr & $1050+35+45$ & $98 \mathrm{ab}$ & $100 \mathrm{a}$ & $0.2 \mathrm{a}$ & $0.9 \mathrm{a}$ \\
\hline
\end{tabular}

${ }^{a}$ Abbreviations: WAE, weeks after crop emergence; PPI, preplant incorporated.

s-metolachlor + halosulfuron, s-metolachlor + imazethapyr, and s-metolachlor + halosulfuron + imazethapyr applied PPI reduced redroot pigweed density $87-98 \%$ and biomass $80-$ 99\% (Table 2).

In other studies, halosulfuron provided $80-81 \%$ control of redroot pigweed [11]. Halosulfuron plus fomesafen POST provided $89-92 \%$ control of redroot pigweed and the tankmix of halosulfuron plus bentazon plus fomesafen also provided 89-91\% control of redroot pigweed [11]. Imazethapyr applied alone or in combination with other herbicides has been shown to reduce redroot pigweed density 69-100\% [13].

3.2.2. Common Ragweed. Trifluralin, s-metolachlor, halosulfuron, imazethapyr, trifluralin + halosulfuron, trifluralin + imazethapyr, trifluralin + halosulfuron + imazethapyr, smetolachlor + halosulfuron, s-metolachlor + imazethapyr, and s-metolachlor + halosulfuron + imazethapyr applied
PPI controlled common ragweed 19-28\%, 30-40\%, 97-99\%, $73-84 \%, 90-96 \%, 78-88 \%, 96-98 \%, 93-98 \%, 78-90 \%$, and 94-98\%, respectively (Table 3). The two- and three-way tankmixes of halosulfuron with trifluralin, s-metolachlor, and imazethapyr were most effective as they provided 90-98\% control of common ragweed.

Trifluralin and s-metolachlor applied PPI had no effect on common ragweed density or biomass but halosulfuron reduced common ragweed density $94 \%$ and biomass $100 \%$ compared to the weedy check (Table 3 ). Trifluralin + halosulfuron, trifluralin + imazethapyr, trifluralin + halosulfuron + imazethapyr, s-metolachlor + halosulfuron, s-metolachlor + imazethapyr, and s-metolachlor + halosulfuron + imazethapyr applied PPI reduced common ragweed density 44-100\% and biomass 56-100\% (Table 3). Halosulfuron applied alone or in tankmix combination with trifluralin, s-metolachlor, and imazethapyr provided the most consistent density 
TABLE 5: Percent control, density, and dry weight of wild mustard in white bean treated with halosulfuron tankmixes applied PPI at Exeter, ON (2011 and 2013). Means followed by the same letter within a column are not significantly different according to Fisher's Protected LSD at $P<0.05^{\mathrm{a}}$.

\begin{tabular}{|c|c|c|c|c|c|}
\hline \multirow{3}{*}{ Herbicide treatment } & \multirow{3}{*}{ Rate (g ai ha ${ }^{-1}$ ) } & \multicolumn{2}{|c|}{ Control } & \multirow{3}{*}{$\begin{array}{l}\text { Density } \\
\# \mathrm{~m}^{-2}\end{array}$} & \multirow{3}{*}{$\begin{array}{c}\text { Dry weight } \\
\mathrm{g} \mathrm{m}^{-2}\end{array}$} \\
\hline & & 4 WAE & 8 WAE & & \\
\hline & & \multicolumn{2}{|c|}{$\%$} & & \\
\hline Weedy check & & $0 \mathrm{~d}$ & $0 \mathrm{~d}$ & $12.6 \mathrm{c}$ & $95.1 \mathrm{c}$ \\
\hline Weed-free check & & $100 \mathrm{a}$ & $100 \mathrm{a}$ & $0.0 \mathrm{a}$ & $0.0 \mathrm{a}$ \\
\hline Trifluralin & 600 & $15 \mathrm{c}$ & $14 \mathrm{c}$ & $12.8 \mathrm{c}$ & $103.4 \mathrm{c}$ \\
\hline s-Metolachlor & 1050 & $35 \mathrm{~b}$ & $12 \mathrm{c}$ & $13.4 \mathrm{c}$ & $122.2 \mathrm{c}$ \\
\hline Halosulfuron & 35 & $100 \mathrm{a}$ & $100 \mathrm{a}$ & $0.1 \mathrm{ab}$ & $0.1 \mathrm{a}$ \\
\hline Imazethapyr & 45 & $97 \mathrm{a}$ & $96 \mathrm{~b}$ & $1.4 \mathrm{~b}$ & $0.7 \mathrm{ab}$ \\
\hline Trifluralin + halosulfuron & $600+35$ & $100 \mathrm{a}$ & $100 \mathrm{a}$ & $0.3 \mathrm{ab}$ & $0.3 \mathrm{ab}$ \\
\hline Trifluralin + imazethapyr & $600+45$ & 99 a & $97 \mathrm{~b}$ & $1.6 \mathrm{~b}$ & $2.2 \mathrm{~b}$ \\
\hline Trifluralin + halosulfuron + imazethapyr & $600+35+45$ & $100 \mathrm{a}$ & $100 \mathrm{a}$ & $0.0 \mathrm{a}$ & $0.0 \mathrm{a}$ \\
\hline s-Metolachlor + halosulfuron & $1050+35$ & $100 \mathrm{a}$ & $100 \mathrm{a}$ & $0.0 \mathrm{a}$ & $0.0 \mathrm{a}$ \\
\hline s-Metolachlor + imazethapyr & $1050+45$ & 98 a & $98 \mathrm{~b}$ & $1.0 \mathrm{ab}$ & $0.3 \mathrm{ab}$ \\
\hline s-Metolachlor + halosulfuron + imazethapyr & $1050+35+45$ & $100 \mathrm{a}$ & $100 \mathrm{a}$ & $0.0 \mathrm{a}$ & $0.0 \mathrm{a}$ \\
\hline
\end{tabular}

${ }^{a}$ Abbreviations: WAE, weeks after crop emergence; PPI, preplant incorporated.

TABLE 6: Percent control, density, and dry weight of green foxtail in white bean treated with halosulfuron tankmixes applied PPI at Exeter, ON (2011-2013). Means followed by the same letter within a column are not significantly different according to Fisher's Protected LSD at $P<0.05^{\mathrm{a}}$.

\begin{tabular}{|c|c|c|c|c|c|}
\hline \multirow{3}{*}{ Herbicide treatment } & \multirow{3}{*}{ Rate $\left(\right.$ g ai ha $^{-1}$ ) } & \multicolumn{2}{|c|}{ Control } & \multirow{3}{*}{$\begin{array}{l}\text { Density } \\
\# \mathrm{~m}^{-2}\end{array}$} & \multirow{3}{*}{$\begin{array}{c}\text { Dry weight } \\
\mathrm{g} \mathrm{m}^{-2}\end{array}$} \\
\hline & & 4 WAE & 8 WAE & & \\
\hline & & \multicolumn{2}{|c|}{$\%$} & & \\
\hline Weedy check & & $0 \mathrm{~g}$ & $0 \mathrm{f}$ & $36.7 \mathrm{c}$ & $38.2 \mathrm{~d}$ \\
\hline Weed-free check & & $100 \mathrm{a}$ & $100 \mathrm{a}$ & $0.0 \mathrm{a}$ & $0.0 \mathrm{a}$ \\
\hline Trifluralin & 600 & $97 \mathrm{bc}$ & $96 \mathrm{bc}$ & $1.7 \mathrm{a}$ & $1.0 \mathrm{abc}$ \\
\hline s-Metolachlor & 1050 & $95 \mathrm{~cd}$ & $97 \mathrm{~b}$ & $2.6 \mathrm{a}$ & $1.5 \mathrm{abc}$ \\
\hline Halosulfuron & 35 & $53 \mathrm{f}$ & $56 \mathrm{e}$ & $52.5 \mathrm{c}$ & $28.2 \mathrm{~d}$ \\
\hline Imazethapyr & 45 & $82 \mathrm{e}$ & $80 \mathrm{~d}$ & $11.0 \mathrm{~b}$ & $4.5 \mathrm{c}$ \\
\hline Trifluralin + halosulfuron & $600+35$ & $93 \mathrm{~d}$ & $93 c$ & $2.4 \mathrm{a}$ & $2.9 \mathrm{bc}$ \\
\hline Trifluralin + imazethapyr & $600+45$ & $96 \mathrm{bcd}$ & $94 \mathrm{bc}$ & $1.7 \mathrm{a}$ & $0.8 \mathrm{abc}$ \\
\hline Trifluralin + halosulfuron + imazethapyr & $600+35+45$ & $99 \mathrm{~b}$ & $97 \mathrm{~b}$ & $0.6 \mathrm{a}$ & $0.2 \mathrm{ab}$ \\
\hline s-Metolachlor + halosulfuron & $1050+35$ & $95 \mathrm{~cd}$ & $93 c$ & $1.5 \mathrm{a}$ & $0.6 \mathrm{abc}$ \\
\hline s-Metolachlor + imazethapyr & $1050+45$ & $97 \mathrm{bc}$ & $94 \mathrm{bc}$ & $1.1 \mathrm{a}$ & $0.8 \mathrm{abc}$ \\
\hline s-Metolachlor + halosulfuron + imazethapyr & $1050+35+45$ & $98 \mathrm{~b}$ & $96 \mathrm{bc}$ & $1.2 \mathrm{a}$ & $0.8 \mathrm{abc}$ \\
\hline
\end{tabular}

${ }^{a}$ Abbreviations: WAE, weeks after crop emergence; PPI, preplant incorporated.

reduction (83-100\%) and biomass reduction (88-100\%) of common ragweed among herbicide treatments evaluated (Table 3).

In other studies, halosulfuron provided 91-94\% control of common ragweed. Halosulfuron plus fomesafen applied POST provided 93-95\% control of common ragweed [11]. Also, the tankmix of halosulfuron plus bentazon plus fomesafen provided $97-98 \%$ control of common ragweed [11]. Imazethapyr and imazamox have been shown to control common ragweed 76 to $100 \%$ in dry bean [14-16].

3.2.3. Common Lambsquarters. S-metolachlor provided only $63-82 \%$ control of common lambsquarters but trifluralin, halosulfuron, imazethapyr, trifluralin + halosulfuron, trifluralin + imazethapyr, trifluralin + halosulfuron + imazethapyr, s-metolachlor + halosulfuron, s-metolachlor + imazethapyr, and s-metolachlor + halosulfuron + imazethapyr controlled redroot pigweed 94-100\% (Table 4). Similarly, s-metolachlor had no effect on density or biomass of common lambsquarters but other treatments evaluated reduced common lambsquarters density $81-97 \%$ and biomass 76-95\% (Table 4). The two- and three-way tankmixes of halosulfuron with trifluralin, s-metolachlor, and imazethapyr provided 97$100 \%$ control of common lambsquarters.

In other studies, trifluralin applied PPI provided better control of common lambsquarters (83 versus 71\%) compared with s-metolachlor [17]. Halosulfuron plus fomesafen POST provided 55-59\% control of common lambsquarters [11]. The tankmix of halosulfuron plus bentazon plus fomesafen provided $79-82 \%$ control of common lambsquarters [11]. 
Imazethapyr applied alone or in combination with other herbicides has been shown to reduce common lambsquarters density $69-100 \%$ [13]. Imazethapyr and imazamox also have been shown to control common lambsquarters 76 to $100 \%$ in dry bean [14-16].

3.2.4. Wild Mustard. Trifluralin and s-metolachlor provided only $12-35 \%$ control of wild mustard but halosulfuron, imazethapyr, trifluralin + halosulfuron, trifluralin + imazethapyr, trifluralin + halosulfuron + imazethapyr, smetolachlor + halosulfuron, s-metolachlor + imazethapyr, and s-metolachlor + halosulfuron + imazethapyr provided 96-100\% control of wild mustard in white bean (Table 5). Halosulfuron applied alone or in two- and three-way tankmixes of halosulfuron with trifluralin, s-metolachlor, and imazethapyr provided $100 \%$ control of wild mustard (Table 5). Trifluralin and s-metolachlor had no effect on density or biomass of wild mustard but other treatments evaluated reduced wild mustard density $87-100 \%$ and biomass $98-100 \%$ (Table 5).

3.2.5. Green Foxtail. Trifluralin, s-metolachlor, halosulfuron, imazethapyr, trifluralin + halosulfuron, trifluralin + imazethapyr, trifluralin + halosulfuron + imazethapyr, s-metolachlor + halosulfuron, s-metolachlor + imazethapyr, and s-metolachlor + halosulfuron + imazethapyr applied PPI controlled green foxtail as much as $97,97,56,82,93,96,99$, 95,97 , and $98 \%$, respectively (Table 6). Halosulfuron applied PPI alone had no effect on density or biomass of green foxtail but other treatments evaluated reduced green foxtail density 70-98\% and biomass $88-99 \%$ (Table 6). The twoand three-way tankmixes of halosulfuron with trifluralin, s-metolachlor, and imazethapyr provided 93-98\% control of green foxtail. In other studies, the control of some grasses such as E. crus-galli increased from $58-96 \%$ to $98 \%$ when s-metolachlor or trifluralin was applied PPI in combination with broadleaf herbicides such as imazethapyr [2].

\section{Conclusions}

Trifluralin, s-metolachlor, halosulfuron, imazethapyr, trifluralin + halosulfuron, trifluralin + imazethapyr, trifluralin + halosulfuron + imazethapyr, s-metolachlor + halosulfuron, s-metolachlor + imazethapyr, and s-metolachlor + halosulfuron + imazethapyr applied PPI caused minimal and transient injury in white bean. Trifluralin applied PPI at $600 \mathrm{~g}$ ai ha $^{-1}$ provided excellent full season control of redroot pigweed, common lambsquarters, and green foxtail and poor control of common ragweed and wild mustard. Smetolachlor applied PPI at $1050 \mathrm{~g}$ ai ha ${ }^{-1}$ provided excellent full season control of green foxtail, good control of redroot pigweed, fair control of common lambsquarters, and poor control of common ragweed and wild mustard. Halosulfuron applied PPI at $35 \mathrm{~g}$ ai ha ${ }^{-1}$ provided excellent full season control of common lambsquarters, redroot pigweed, common ragweed, and wild mustard and fair control of green foxtail. Imazethapyr applied PPI at $45 \mathrm{~g}_{\text {ai ha }}{ }^{-1}$ provided excellent full season control of redroot pigweed, common lambsquarters, and wild mustard and good control of common ragweed and green foxtail. The two- and three-way tankmixes of halosulfuron with other herbicides evaluated provided good to excellent full season control of redroot pigweed, common ragweed, common lambsquarters, wild mustard, and green foxtail. Generally, white bean yields reflected the level of weed control. Based on these results, halosulfuron applied PPI in two-way or three-way tankmix with trifluralin, smetolachlor, and/or imazethapyr can be used safely for the control of annual grass and broadleaf weeds in white bean.

\section{Conflict of Interests}

The authors have no conflict of interests to report.

\section{Acknowledgment}

Funding for this project was provided by the Ontario Bean Growers and the GF2 program of Agricultural Adaptation Council.

\section{References}

[1] K. Kulasekera, "Estimated area, yield, production and farm value of specified field crops, Ontario, 2001-2013, (metric units)," 2014, http://www.omafra.gov.on.ca/english/stats/crops/ estimate_metric.htm.

[2] N. R. Arnold, W. M. Murray, J. E. Gregory, and D. Smeal, "Weed control in pinto beans (Phaseolus vulgaris) with imazethapyr combinations," Weed Technology, vol. 7, pp. 361-364, 1993.

[3] T. A. Bauer, K. A. Renner, D. Penner, and J. D. Kelly, "Pinto bean (Phaseolus vulgaris) varietal tolerance to imazethapyr," Weed Science, vol. 43, no. 3, pp. 417-424, 1995.

[4] R. E. Blackshaw and G. Saindon, "Dry bean (Phaseolus vulgaris) tolerance to imazethapyr," Canadian Journal of Plant Science, vol. 76, no. 4, pp. 915-919, 1996.

[5] C. P. Urwin, R. G. Wilson, and D. A. Mortensen, "Response of dry edible bean (Phaseolus vulgaris) cultivars to four herbicides," Weed Technology, vol. 10, no. 3, pp. 512-518, 1996.

[6] R. G. Wilson and S. D. Miller, "Dry edible bean (Phaseolus vulgaris) responses to imazethapyr," Weed Technology, vol. 5, pp. 22-26, 1991.

[7] Ontario Ministry of Agriculture and Food and Rural Affairs (OMAFRA), Guide to Weed Control, Publication 75, Ontario Ministry of Agriculture and Food and Rural Affairs (OMAFRA), Toronto, Canada, 2013.

[8] S. A. Senseman, Herbicide Handbook, Weed Sci. Soc. Am., Champaign, Ill, USA, 9th edition, 2007.

[9] N. Soltani, C. Shropshire, and P. H. Sikkema, "Response of dry beans to halosulfuron applied postemergence," Canadian Journal of Plant Science, vol. 92, no. 4, pp. 723-728, 2012.

[10] C. L. Stewart, R. E. Nurse, C. Gillard, and P. H. Sikkema, “Tolerance of adzuki bean to preplant-incorporated, pre-emergence, and post-emergence herbicides in Ontario, Canada," Weed Biology and Management, vol. 10, no. 1, pp. 40-47, 2010.

[11] N. Soltani, R. E. Nurse, and P. H. Sikkema, "Weed management in white beans with postemergence herbicide tankmixes," Canadian Journal of Plant Science, vol. 93, no. 4, pp. 669-674, 2013.

[12] B. D. Silvey, W. E. Mitchem, A. W. Macrae, and D. W. Monks, "Snap bean (Phaseolus vulgaris) tolerance to halosulfuron PRE, 
POST, or PRE followed by POST,' Weed Technology, vol. 20, no. 4, pp. 873-876, 2006.

[13] D. Wall, "Bentazon tank-mixtures for improved redroot pigweed (Amaranthus retroflexus) and common lambsquarters (Chenopodium album) control in navy bean (Phaseolus vulgaris)," Weed Technology, vol. 9, no. 3, pp. 610-616, 1995.

[14] O. C. Burnside, W. H. Ahrens, B. J. Holder, M. J. Weins, M. M. Johnson, and E. A. Ristau, "Efficacy and economics of various mechanical plus chemical weed control systems in dry beans (Phaseolus vulgaris)," Weed Technology, vol. 8, no. 2, pp. 238244, 1994.

[15] M. J. Vangessel and P. Westra, "Economics and efficacy of postemergence spurred anoda (Anoda cristata) control in pinto beans (Phaseolus vulgaris)," Weed Technology, vol. 11, no. 2, pp. 329-334, 1997.

[16] R. G. Wilson Jr., "Response of dry bean and weeds to fomesafen and fomesafen tank mixtures," Weed Technology, vol. 19, no. 1, pp. 201-206, 2005.

[17] P. H. Sikkema, N. Soltani, C. Shropshire, and T. Cowan, "Tolerance of white beans to postemergence broadleaf herbicides," Weed Technology, vol. 18, no. 4, pp. 893-901, 2004. 


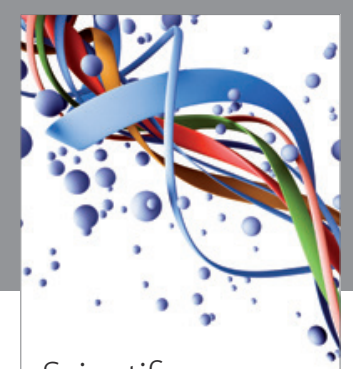

Scientifica
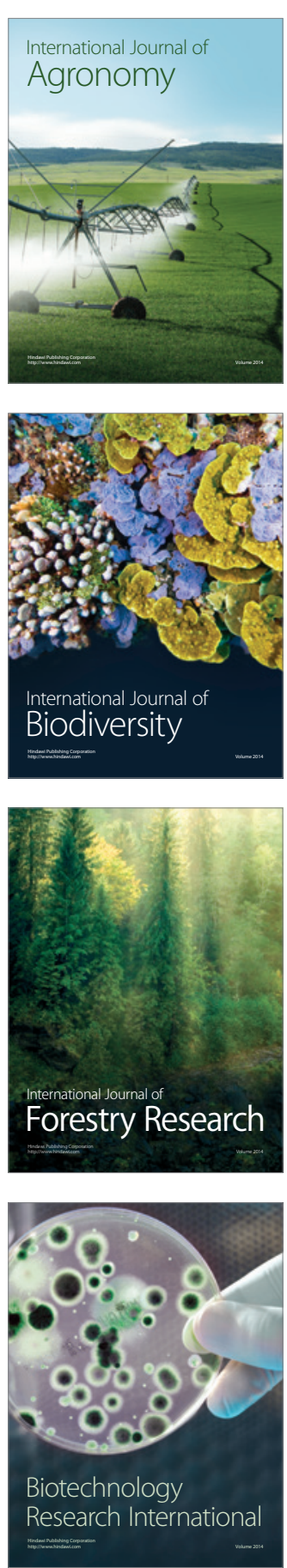
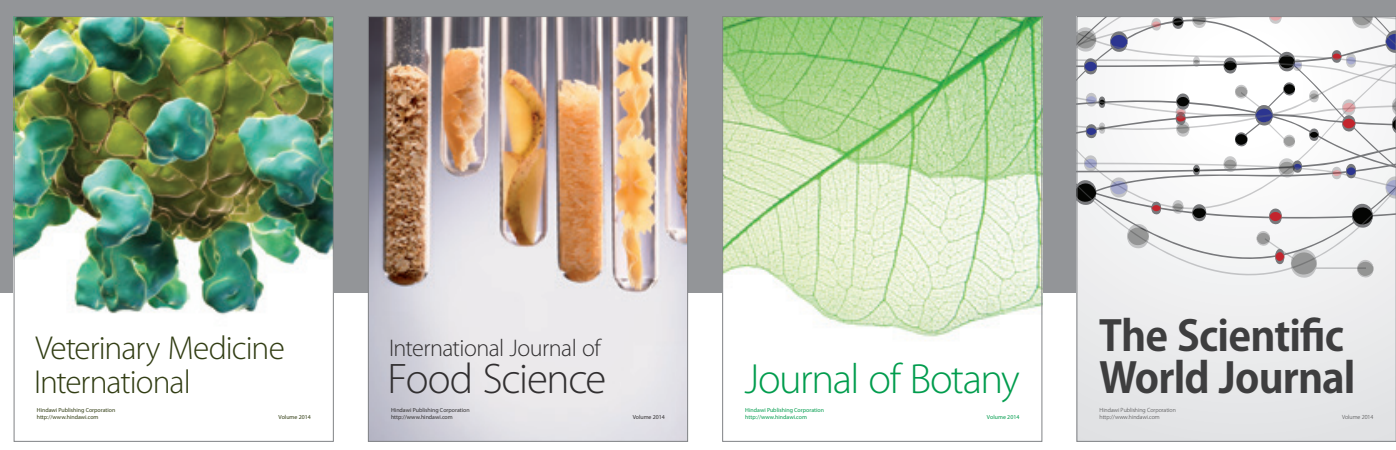

The Scientific World Journal
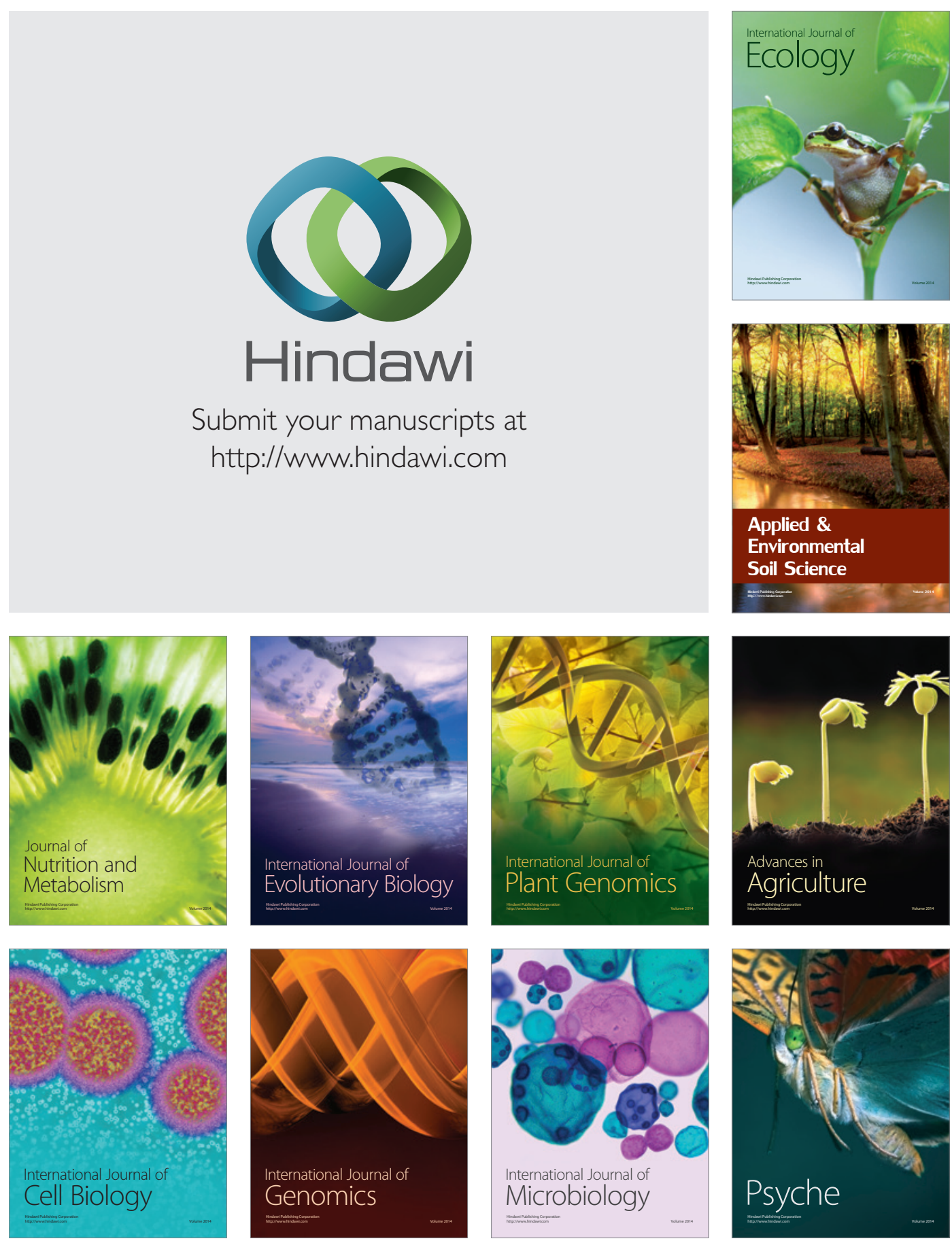